\title{
Terminal Deoxynucleotidyl Transferase Commonly Expresses in Germ Cell Tumors: Evaluation on a Large Series from Multiple Centers
}

This article was published in the following Dove Press journal:

International Journal of General Medicine

Jun Zhou',*

Suying Wang ${ }^{2, *}$

Lun Zhu ${ }^{3}$

Luting Zhou'

Hong Zeng ${ }^{4}$

Yongli Gan ${ }^{2}$

Chaofu Wang (D)

'Department of Pathology, Ruijin Hospital, Shanghai Jiaotong University School of Medicine, Shanghai, People's Republic of China; ${ }^{2}$ Department of Pathology, Ningbo Clinical and Pathological Diagnostic Center, Ningbo Zhejiang, People's Republic of China;

${ }^{3}$ Department of Pathology, Sir Run Run Shaw Hospital, School of Medicine, Zhejiang University, Hangzhou, Zhejiang, People's Republic of China; ${ }^{4}$ Department of Pathology, Sun Yat-Sen Memorial Hospital, Sun Yat-Sen University, Guangzhou, Guangdong, People's Republic of China

*These authors contributed equally to this work

\begin{abstract}
Aim: The concrete features of expression of terminal deoxynucleotidyl transferase (TdT) are needed to be revealed in male and female germ cell tumors (GCTs).

Methods: TdT immunostaining was performed in 195 GCTs, and the tumor and/or tumorous components included seminomas, germ cell neoplasias in situ (GCNISs), dysgerminomas, embryonal carcinomas (ECs), extragonadal germinomas, yolk sac tumors (YSTs), teratomas, and spermatocytic tumors. Twenty-one sex cord-stromal tumors were also added. Expression of the classical germ cell tumor markers (PLAP, OCT4, SALL4, CD117, and D2-40) was compared to that of TDT.

Results: Nearly all (tumors or tumorous components) seminomas (99\%, 107/108), GCNISs $(98 \%, 51 / 52)$, dysgerminomas $(94 \%, 17 / 18)$, ECs $(100 \%, 15 / 15)$, and extragonadal germinomas $(100 \%, 11 / 11)$ were positive for TdT. None of the cells in YSTs $(0 / 38)$, teratomas $(0 /$ $19)$, spermatocytic tumors $(0 / 1)$, or sex cord-stromal tumors $(0 / 21)$ were immunoreactive for TdT staining. The normal testicular and ovarian gonadal tissues were also negative for TdT. However, TdT presented with significant loss of antigen immunoreactivity in the paraffinembedded tissues older than 3 years, giving rise to weak or moderate staining in a subset of cases. The expressions of TdT showed no significances with PLAP, OCT4, SALL4, CD117, and D2-40 during the diagnosis of the most GCTs $(P>0.05)$, except for with PLAP, SALL4, or CD117 in YST $(P=0.000$ each), and D117 $(P=0.000)$ or D2-40 $(P=0.006)$ in ECs.

Conclusion: Our findings further verify that TdT can serve as a new GCT marker for seminomas, GCNISs, dysgerminomas, ECs, and extragonadal germinomas, with a highly positive rate. Awareness of TdT positivity in GCTs contributes to the prevention of erroneous diagnoses, particularly in the setting of core needle biopsies. To determine the properties where TdT staining may not be apparent in some old archived paraffin-embedded tissues, one could circumvent the potential misinterpretations of false-negative immunohistochemistry results.
\end{abstract}

Keywords: germ cell tumors, immunohistochemistry, TdT, pathological diagnosis

\section{Introduction}

Germ cell tumors (GCTs) are a group of tumors presumably arising from primordial germ cells, frequently involving the testis or ovary, although they can arise in extragonadal sites. ${ }^{1,2}$ Ninety percent of primary testicular tumors and $30 \%$ of primary ovarian tumors are of germ cell origin. ${ }^{3,4}$ Malignant GCTs are rare, and
Department of Pathology, Ruijin Hospital, Shanghai Jiaotong University School of Medicine, 2st Ruijin Road, Huangpu

District, Shanghai, People's Republic of China

Email wangchaofu@I26.com

International Journal of General Medicine 2021:|4 I| 19-129 
the vast majority of them occur in adolescents or young adults. ${ }^{5}$ GCTs are characterized by distinct heterogeneity, which impacts their biological behavior and clinical management. ${ }^{6}$ This complex nature is reflected in the updated WHO classification of testicular cancer. ${ }^{7}$

Terminal deoxynucleotidyl transferase (TdT), a nontemplate directed DNA polymerase, is highly expressed in immature $\mathrm{B}$ or $\mathrm{T}$ lymphoid cells as well as lymphoblastic lymphoma/leukemia (LBL). ${ }^{8}$ Although positive in a small fraction of reactive lymphoid proliferation cases and some acute myeloid leukemias, TdT is generally considered a reliable immunostaining marker for the recognition of LBL, with high sensitivity and specificity. ${ }^{9-11}$ However, some nonlymphoid tumors tagged with $\mathrm{TdT}$ exist, including $73 \%$ of Merkel cell carcinomas, myoepithelial cells, few small cell lung carcinomas, and normal and neoplastic sebaceous cells. ${ }^{12-16}$ Recently, we encountered a case of mediastinal seminoma demonstrating diffusely TdT-positive nuclei. Alexander Brobeil et al reported that seminoma (10/10) and germ cell neoplasia in situ (GCINS) (2/2) were positive for TdT. ${ }^{17}$ However, the bona fide expression spectrum of $\mathrm{TdT}$ in germ or germ-related disease is still cryptic.
Here, we performed TdT immunostaining in various germ cell tumors and an additional 20 cases of sex cordstromal tumors in both male and female patients to discuss the expanded application of TdT and potential diagnostic pitfalls.

\section{Patients and Methods \\ Case Selection}

The informed consent from all of the involved patients was obtained that their tissues can be used in our research. This study was approved by the institutional review boards of Ruijin Hospital, Shanghai Jiaotong University School of Medicine, Sir Run Run Shaw Hospital, and Ningbo Clinical and Pathological Diagnostic Center (ID: RJBLK20180901). The detailed personal information of each patient sample was used according to the principles of the Declaration of Helsinki. ${ }^{18}$ Of the 195 GCTs and 21 sex cord-stromal tumors (Table 1), 146 cases (55 seminomas, 2 pure GCNISs, 5 dysgerminomas, 2 pure embryonal carcinomas [ECs], 7 mixed GCTs, 11 extragonadal germinomas, 28 YSTs, 15 teratomas, 21 sex cord-stromal tumors) were from the Department of Pathology, Ruijin Hospital, Shanghai Jiaotong University School of

Table I TdT Immunoreactivity in GCTs and Sex Cord-Stromal Tumors

\begin{tabular}{|c|c|c|c|c|c|c|c|c|c|}
\hline \multirow[t]{2}{*}{ Tumor } & \multirow[t]{2}{*}{ No. of Cases } & \multicolumn{5}{|c|}{ Extent of Reactivity* No. (\%) } & \multicolumn{3}{|c|}{ Intensity of Reactivity No. (\%) } \\
\hline & & 0 & I+ & $2+$ & $3+$ & $4+$ & Mild & Moderate & Strong \\
\hline SE & 95 & $0(0)$ & $0(0)$ & $3(3)$ & $8(8)$ & $84(88)$ & $25(26)$ & $14(15)$ & $56(59)$ \\
\hline GCNIS/GCNIS in SE & 41 & $0(0)$ & $0(0)$ & $0(0)$ & $6(15)$ & $35(85)$ & $7(17)$ & $5(12)$ & $29(7 I)$ \\
\hline DG & 16 & I (6) & $0(0)$ & I (6) & $2(13)$ & $12(75)$ & $4(27)$ & $4(27)$ & $7(47)$ \\
\hline EC & 4 & $0(0)$ & $0(0)$ & $0(0)$ & I (25) & $3(75)$ & I (25) & $3(75)$ & $0(0)$ \\
\hline EG & 11 & $0(0)$ & $0(0)$ & $0(0)$ & $0(0)$ & II (100) & $0(0)$ & $0(0)$ & II (100) \\
\hline YST & 28 & $28(100)$ & $0(0)$ & $0(0)$ & $0(0)$ & $0(0)$ & $0(0)$ & $0(0)$ & $0(0)$ \\
\hline ST & 1 & I $(100)$ & $0(0)$ & $0(0)$ & $0(0)$ & $0(0)$ & $0(0)$ & $0(0)$ & $0(0)$ \\
\hline MGCT & 23 & & & & & & & & \\
\hline \multicolumn{10}{|l|}{ Components of } \\
\hline SE & 13 & I (8) & $0(0)$ & $0(0)$ & $2(17)$ & $10(75)$ & $3(27)$ & $3(27)$ & $7(45)$ \\
\hline GCNIS & 11 & I (0) & $0(0)$ & $0(0)$ & $0(0)$ & 10 (91\%) & $I(10)$ & $4(40)$ & $5(50)$ \\
\hline DG & 2 & $0(0)$ & $0(0)$ & $0(0)$ & I (50) & I (50) & I (50) & $0(0)$ & I (50) \\
\hline EC & 11 & $0(0)$ & $0(0)$ & $0(0)$ & $2(18)$ & $9(82)$ & $5(45)$ & I (9) & $5(45)$ \\
\hline YST & 10 & $10(100)$ & $0(0)$ & $0(0)$ & $0(0)$ & $0(0)$ & $0(0)$ & $0(0)$ & $0(0)$ \\
\hline Teratoma & 4 & $4(100)$ & $0(0)$ & $0(0)$ & $0(0)$ & $0(0)$ & $0(0)$ & $0(0)$ & $0(0)$ \\
\hline Trophoblast & 6 & $6(100)$ & $0(0)$ & $0(0)$ & $0(0)$ & $0(0)$ & $0(0)$ & $0(0)$ & $0(0)$ \\
\hline Teratoma & 15 & $15(100)$ & $0(0)$ & $0(0)$ & $0(0)$ & $0(0)$ & $0(0)$ & $0(0)$ & $0(0)$ \\
\hline SCST & 21 & $21(100)$ & $0(0)$ & $0(0)$ & $0(0)$ & $0(0)$ & $0(0)$ & $0(0)$ & $0(0)$ \\
\hline
\end{tabular}

Notes: $* 0$, no staining; I+, fewer than 30 positive; $2+, 31 \%$ to $60 \%$ positive; $3+, 61-90 \%$ positive; 4 , more than $90 \%$ positive.

Abbreviations: DG, dysgerminoma; EC, embryonal carcinoma; EG, extragonadal germinoma; GCNIS, germ cell neoplasias in situ; MGCT, mixed germ cell tumor; SCST, sex cord-stromal tumor; SE, seminoma; ST, spermatocytic tumor; YST, yolk sac tumor. 
Medicine (Shanghai, China); 63 cases (40 seminomas, 7 dysgerminomas, 15 mixed GCTs, 1 spermatocytic tumor) were from the Department of Pathology, Ningbo Clinical and Pathological Diagnostic Center, (Ningbo, China); and 7 cases (4 dysgerminomas, 2 pure ECs, 1 mixed GCTs) were from Sir Run Run Shaw Hospital, School of Medicine, Zhejiang University between January 1, 2010, and June 30, 2018. Another 10 cases of the nonneoplastic testis and ovary lesions from Ruijin Hospital were also included as controls. Histological and relevant immunohistochemical slides (PLAP, OCT4, SALL4, CD117, and D2-40) were reviewed by experienced pathologists (J. Z. and C.W.) to confirm the diagnosis and select the representative sections for TdT immunostaining. ${ }^{19-21}$

\section{Immunohistochemistry Analysis}

All samples were stained for TdT. PLAP, OCT4, SALL4, CD17, and D2-40 were also stained in some old archived tumor tissues or tissues devoid of available immunohistochemical information. $^{21}$ Four-micron-thick sections were taken from $10 \%$ formalin-fixed and paraffin-embedded tissue blocks, as described previously in literature. ${ }^{15,22}$ Then the immunohistochemical staining was performed using a Dako automated immunohistochemistry system (Dako, Carpinteria, CA, US) according to the manufacturer's protocol. ${ }^{23}$ All of the primary antibodies were commercially available including TdT (EP266, prediluted; Dako, Carpinteria, CA, US), PLAP (EP194, 1:100; ZSGB-Bio, Beijing, China), OCT4 (N1NK, prediluted; Dako, Carpinteria, CA, US), SALL4 (6E3, 1:50; ZSGB-Bio, Beijing, China), CD117 (YR145, prediluted, MXB Biotech, Fuzhou, Fujian, China) and D2-40 (D2-40, prediluted, Dako, Carpinteria, CA, US). Detection of antibody binding was obtained using the universal immunoperoxidase polymer method (Envision-Kit; Dako, Carpinteria, CA, US). Appropriate positive (thymus) and negative controls (fibroblasts) were used. ${ }^{15}$ For some unsatisfactory staining results due to long-term archiving, we selected another representative tissue block and optimized antigen retrieval and coloration time; another Bond automated immunohistochemistry system (Leica Microsystems, Wetzlar, Germany) was also used. Immunoreactivity was evaluated semiquantitatively according to the percentage of immunoreactive tumor cells: 0 , no staining; $1+$, fewer than $30 \%$ positive; $2+, 31 \%$ to $60 \%$ positive; $3+, 61-90 \%$ positive; $4+$, more than $90 \%$ positive. More than $3+$ refers to "diffusely positive", whereas $1+$ or $2+$ refers to "focally positive". The intensity of staining was graded as mild, moderate, or strong.

\section{Statistical Analysis}

Statistical analyses were performed to compare TdT staining to that of PLAP, OCT4, SALL4, CD117, D2-40 in the components of GCTs (Table 3). Chi-Square test was used to compare TdT staining to that of seminomas and GCNISs. Fisher exact test was used to compare TdT staining to that of dysgerminomas, Embryonal carcinomas, Embryonal carcinomas, and Yolk sac tumors. A $P<0.05$ is considered statistically significant. The statistical analysis was performed with SPSS v. 22.0 (IBM SPSS Statistics Inc., Chicago, US).

\section{Results}

\section{TdT in Pure Seminomas and Seminomatous Components in Mixed GCTs $(\mathrm{N}=108)$}

Ninety-five pure seminomas (median age 37.5 y., range: 21 to $70 \mathrm{y}$.) and 13 seminomatous components in mixed GCTs (median age 28 y., range: 25 to 35 y.) were stained for TdT. The former included 91 primary testicular tumors, 2 seminomas secondary to cryptorchidism, and 2 metastatic cases (involving retroperitoneal areas and left supraclavicular lymph nodes, Figure 1A). As depicted in Table 1, 107 cases of pure seminomas and seminomatous components $(99 \%, 107 / 108)$ were positive for TdT staining in over $60 \%$ of tumor cells (pure seminomas, $88 \%, 84 / 95$; mixed GCTs, 77\%, 10/13); of which, the positive proportion of the tumor cells among the majority was over $90 \%$ (Table 1; Figure 1B and C). Only a single case of mixed GCTs stored over 6 years was negative for TdT. The staining intensity varied from mild to strong (mild, 26\%, 28/107; moderate, 14\%, 15/107; strong, 59\%, 63/107) (Table 1) among the TdT-positive cases. Antigen loss due to long-term preservation is one of the potential causes of weak positivity for TdT (Figure 1D). However, 98\% of cases filed in the most recent 3 years demonstrated strong uniform reactivity in over $90 \%$ of tumor cells (Table 2 ).

\section{TdT in GCNIS Components ( $\mathrm{N}=52)$}

Cases came from 41 seminomas (median age $35 \mathrm{y}$., range: 21 to $63 \mathrm{y}$.), 11 mixed GCTs with superimposed GCNIS components (median age 27 y., range: 23 to 35 y.) and 2 male pseudohermaphroditism cases with pure GCNIS (age 14 and $28 \mathrm{y}$ ) (Figure 2A). TdT was positive in almost every tumor cell in all GCNISs except for 1 case $(98 \%, 51 / 52)$. In total, $85 \%(35 / 41)$ of GCNISs in seminomas and $100 \%$ of GCNISs in mixed GCTs were diffusely positive for TdT 

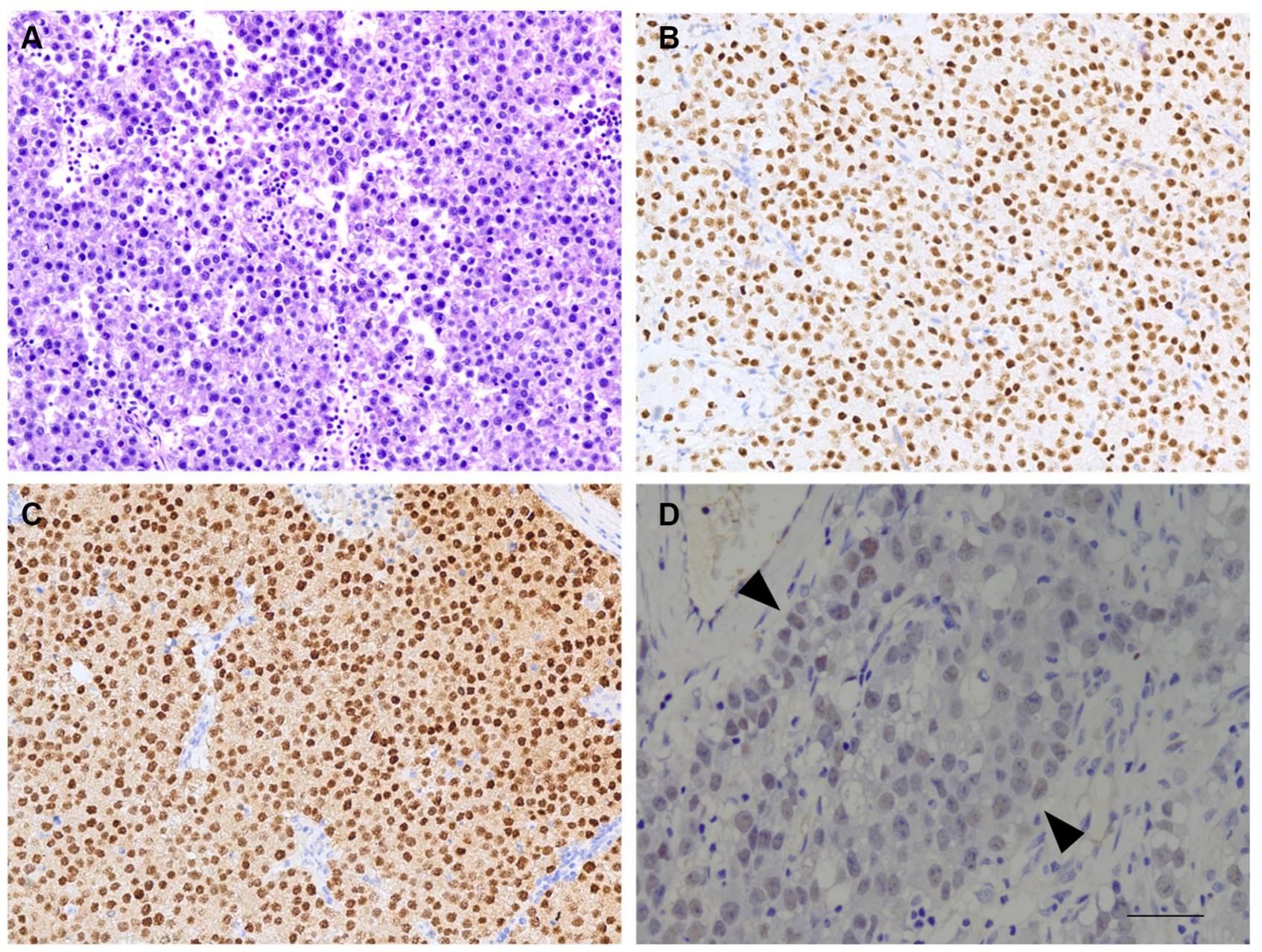

Figure I Immunohistochemical staining of TdT in seminomas. Seminomas are characterized by diffuse sheets or nests of tumor cells separated with lymphocytic infiltration; tumor cells have relatively uniform and large nuclei, prominent nucleoli, and abundant clear or eosinophilic cytoplasm (A). Tumor cells were diffusely and strongly positive for TdT (B) and confirmed by OCT4 (C). Old archived tissues may lose TdT antigen to varying degrees and therefore give a misinterpretation of "negative" staining for TdT (D,

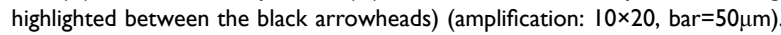

(Table 1; Figure 2B and C). The intensity of tumor cells was mild $(15 \%, 8 / 52)$, modest $(19 \%, 10 / 52)$, or strong $(65 \%, 34 /$ 52) (Table 1). Almost all GCNIS components $(97 \%, 29 / 30)$ in the past 3 years were diffusely and strongly positive for TdT (Table 2).

\section{TdT in Pure Dysgerminomas and Dysgerminomatous Components $(\mathrm{N}=18)$}

There were 16 pure dysgerminomas (median age $22.5 \mathrm{y}$, range: 12 to $40 \mathrm{y}$.) and 2 dysgerminomatous components in mixed GCTs (35 y., 38 y.) (Figure 3A). Fifteen pure dysgerminomas $(94 \%, 15 / 16)$ and 2 dysgerminomatous components $(100 \%, 2 / 2)$ were positive for TdT (Table 1 ; Figure $3 \mathrm{~B}$ and $\mathrm{C}$ ). One pure ovarian dysgerminoma (18 y.) was negative. Seventy-six percent of pure dysgerminoma and dysgerminomatous components were diffusely positive for TdT (Table 1). The intensity of tumor cells was mild $(29 \%, 5 / 17)$, modest $(24 \%, 4 / 17)$, or strong $(47 \%, 8 / 17)$ (Table 1). Eighty-eight percent (7/8) of dysgerminomatous components were strongly positive in the past 3 years (Table 2).

\section{TdT in ECs and EC Components $(\mathrm{N}=15)$}

Four pure ECs (4 primary testicular, mean 26 y. range: 22 to 32 y.; 1 right metastatic supraclavicular ECs, 30 y.) and 11 testicular EC components (median 26 y., range: 23 y. to $47 \mathrm{y}$.) and 1 ovarian EC (38 y.) in mixed GCTs were included (Figure 4A). All ECs and EC components expressed TdT, where diffusely positive cases accounted for $80 \%(12 / 15)$ (Table 1; Figure 4B and C). However, only $33 \%(5 / 15)$ showed strong staining, and $66 \%$ of cases demonstrated mild $(40 \%, 6 / 15)$ or moderate $(27 \%, 4 / 15)$ reactivity (Table 1$)$. Even if retained in the past 3 years, the strongly positive components comprised only approximately 45\% (5/11) (Table 2). 
Table 2 TdT Immunoreactivity in GCTs in the Most Recent 3 Years

\begin{tabular}{|c|c|c|c|c|c|c|c|c|c|}
\hline \multirow[t]{2}{*}{ Tumor } & \multirow[t]{2}{*}{ No. of Cases } & \multicolumn{5}{|c|}{ Extent of Reactivity* No. (\%) } & \multicolumn{3}{|c|}{ Intensity of Reactivity } \\
\hline & & 0 & $1+$ & $2+$ & $3+$ & $4+$ & Mild & Moderate & Strong \\
\hline SE & 50 & $0(0)$ & $0(0)$ & $0(0)$ & I (2) & $48(98)$ & $I(2)$ & I (2) & $48(96)$ \\
\hline GCNIS or GCNIS in SE & 26 & I (0) & $0(0)$ & $0(0)$ & $0(0)$ & $25(96)$ & $0(0)$ & $0(0)$ & $25(100)$ \\
\hline DG & 8 & $0(0)$ & $0(0)$ & $0(0)$ & $I(13)$ & $7(88)$ & $I(13)$ & $0(0)$ & $7(88)$ \\
\hline EC & 4 & $0(0)$ & $0(0)$ & $0(0)$ & I (25) & $3(75)$ & I (25) & $3(75)$ & $0(0)$ \\
\hline EG & 9 & $0(0)$ & $0(0)$ & $0(0)$ & $0(0)$ & $9(100)$ & $0(0)$ & $0(0)$ & $9(100)$ \\
\hline MGCT & 11 & & & & & & & & \\
\hline \multicolumn{10}{|l|}{ Components of } \\
\hline SE & 9 & $0(0)$ & $0(0)$ & $0(0)$ & I (II) & $8(89)$ & $0(0)$ & I (II) & $8(89)$ \\
\hline GCNIS & 4 & $0(0)$ & $0(0)$ & $0(0)$ & $0(0)$ & $4(100)$ & $0(0)$ & $0(0)$ & $4(100)$ \\
\hline DG & 9 & $0(0)$ & $0(0)$ & $0(0)$ & $0(0)$ & $9(100)$ & $0(0)$ & $\mathrm{I}(\mathrm{II})$ & $8(89)$ \\
\hline EC & 7 & $0(0)$ & $0(0)$ & $0(0)$ & I (I4) & $6(86)$ & $2(29)$ & $0(0)$ & $5(7 I)$ \\
\hline
\end{tabular}

Notes: ${ }^{*} 0$, no staining; $1+$, fewer than 30 positive; $2+, 31 \%$ to $60 \%$ positive; $3+, 61-90 \%$ positive; 4 , more than $90 \%$ positive.

Abbreviations: DG, dysgerminoma; EC, embryonal carcinoma; EG, extragonadal germinoma; GCNIS, germ cell neoplasias in situ; MGCT, mixed germ cell tumor; $\mathrm{SE}$, seminoma.

Table 3 Comparation of TdT Staining to That of PLAP, OCT4, SALL4, CDII7, D2-40 in GCTs (Chi-Square/Fisher Exact Test)

\begin{tabular}{|c|c|c|c|c|c|c|c|c|c|c|c|}
\hline \multirow[t]{2}{*}{ Component } & \multirow[t]{2}{*}{ TdT Positive } & \multicolumn{2}{|c|}{ PLAP Positive } & \multicolumn{2}{|c|}{ OCT4 Positive } & \multicolumn{2}{|c|}{ SALL4 Positive } & \multicolumn{2}{|c|}{ CDII7 Positive } & \multicolumn{2}{|c|}{ D2-40 Positive } \\
\hline & & No.(\%) & $P$ & No.(\%) & $P$ & No.(\%) & $P$ & No.(\%) & $P$ & No.(\%) & $P$ \\
\hline SE $(N=108)$ & 107 (99) & $105(97)$ & 0.614 & $105(97)$ & 0.614 & 105 (97) & 0.614 & $104(96)$ & 0.365 & 107 (99) & 1.00 \\
\hline GCNIS (N=52) & $52(100)$ & $52(100)$ & 1.00 & $51(98)$ & 1.00 & $52(100)$ & 1.00 & $50(100)$ & 1.00 & $52(100)$ & 1.00 \\
\hline $\mathrm{DG}(\mathrm{N}=18)$ & $17(94)$ & $17(94)$ & 1.00 & $18(100)$ & 1.00 & $18(100)$ & 1.00 & $17(94)$ & 1.00 & $18(100)$ & 1.00 \\
\hline$E C(N=15)$ & $15(100)$ & $13(87)$ & 0.483 & $15(100)$ & 1.00 & $15(100)$ & 1.00 & $2(13)$ & 0.000 & $8(53)$ & 0.006 \\
\hline$E G(N=I I)$ & $10(100)$ & $10(100)$ & 1.00 & $10(100)$ & 1.00 & $10(100)$ & 1.00 & $10(100)$ & 1.00 & $10(100)$ & 1.00 \\
\hline YST $(N=38)$ & $0(0)$ & $12(33)$ & 0.000 & $0(0)$ & 1.00 & $38(100)$ & 0.000 & $18(38)$ & 0.000 & $0(0)$ & 1.00 \\
\hline
\end{tabular}

Abbreviations: DG, dysgerminoma; EC, embryonal carcinoma; EG, extragonadal germinoma; GCNIS, germ cell neoplasias in situ; SE, seminoma; YST, yolk sac tumor.

\section{TdT in Extragonadal Germinomas $(\mathrm{N}=\mathrm{II})$}

Eleven extragonadal germinomas were composed of one female (18 y.) and 10 males (median 19 y., range: 13 y. to 38 y.) Six samples involved the sellar region (Figure 5A1), 1 developed in the pineal area, 2 occurred in the thalamus, and 2 arose in the mediastinum (Figure 5B1). One of the latter cases metastasized to the right cervical region (Figure 5C1). All of the cases were diffusely and strongly positive for TdT both in the last 3 years and in the earlier years (Tables 1 and 2; Figure 5A2-C2).
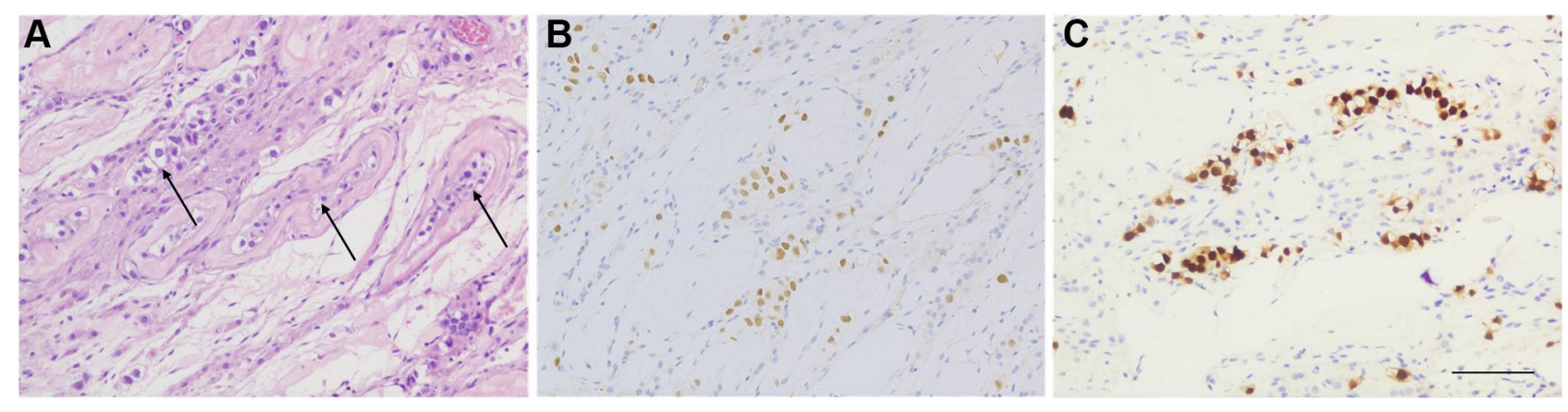

Figure 2 Immunohistochemical staining in GCNIS. GCNIS features large atypical tumor cells located (arrow) on the periphery of seminiferous tubules with prominent nucleoli and abundant clear cytoplasm (A). The tumor cells were strongly positive for TdT (B). These GCNIS cells are confirmed by OCT4 (C) (amplification: I0×20, bar $=50 \mu \mathrm{m})$. 

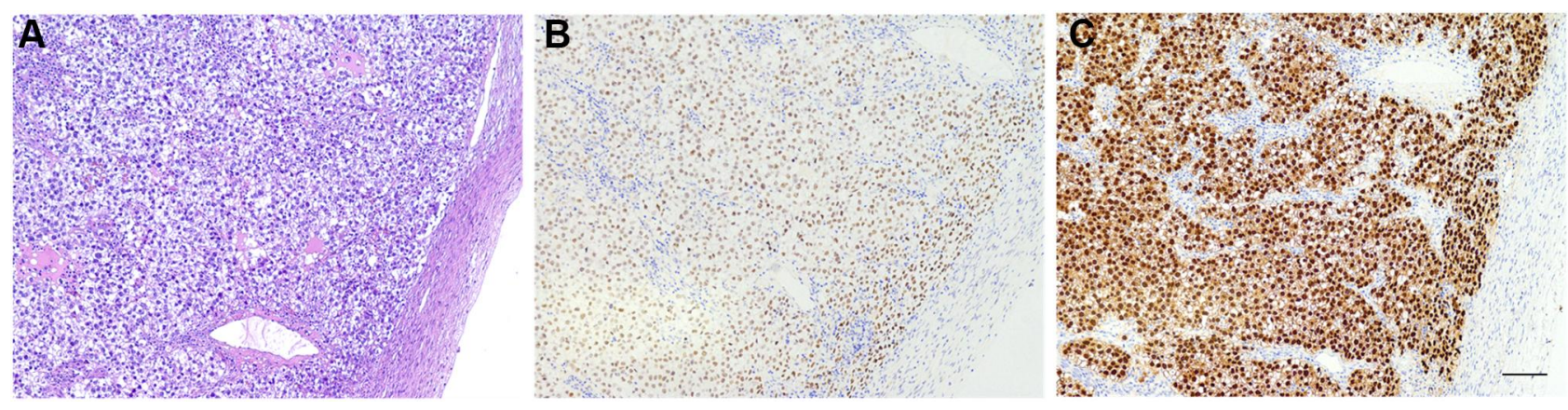

Figure 3 Immunohistochemical staining in dysgerminoma. Dysgerminoma presents with sheets of relatively uniform tumor cells with intervening thin fibrous septa and small

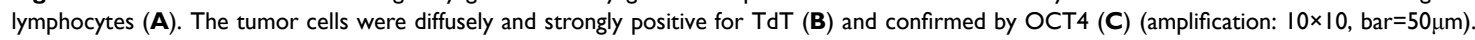

\section{TdT in YSTs and YST Components $(\mathrm{N}=38)$}

Thirteen testicular YSTs, including 8 prepubertal types (median 11.5 mo., range: 8 to 24 mo.) and 5 postpubertal types (median $22 \mathrm{y}$., range: 18 to $52 \mathrm{y}$.), and 6 YST components in testicular mixed GCTs (median 32 y., range: 23 to $47 \mathrm{y}$.) were collected. Besides, 15 ovarian YSTs (median 19.5 y., range: 5 to $31 \mathrm{y}$.) and 4 YST components in ovarian mixed GCTs were also analyzed. All YST components $(100 \%, 38 / 38)$ were completely negative for TdT (Table 1).

\section{TdT in Teratomas and Teratomatous Components $(\mathrm{N}=19)$}

A total of 19 teratomas or teratomatous components were investigated, including 1 testicular prepubertal teratoma (12 mo.), 1 postpubertal (26 у.) pure teratoma, 2 teratomatous components in testicular mixed GCTs (both 28 y.), 5 ovarian mature (median $26 \mathrm{y}$, range: 10 to $51 \mathrm{y}$.) and 7 immature ovarian teratomas (median 23.5, range: 16 to $26 \mathrm{y}$.), as well as 3 teratomatous components in ovarian mixed GCTs $(5,29$, $35 \mathrm{y}$.). All teratomatous components (19/19) were negative for TdT (Table 1).

\section{TdT in Spermatocytic Tumors $(\mathrm{N}=\mathrm{I})$}

One testicular spermatocytic tumor $(58 \mathrm{y}$.) was negative for TdT (Table 1).

\section{TdT in Sex Cord-Stromal Tumors ( $\mathrm{N}=2 \mathrm{I})$ and Normal Gonadal Tissues}

Twenty-one sex cord-stromal tumors including 3 testicular Sertoli cell tumors $(27,27,26$ y.), 2 ovarian Sertoli-Leydig cell tumors $(24,27$ y.), 1 testicular granulosa cell tumor $(63 \mathrm{y}$.$) , and 15$ granulosa cell tumors (median $48 \mathrm{y}$., range: 30 to $67 \mathrm{y}$.) were included. All cells of sex cord-stromal tumors were negative for TdT (Table 1).

TdT staining was performed in 3 normal testicular and 2 normal ovarian tissues. Nonneoplastic cells in some testicular and ovarian GCTs available were also evaluated. All of these cells (spermatogenic cells, 47/47; Sertoli cells $49 / 49$, Leydig cells $8 / 8$, cells of rete testis $15 / 15$, testicular efferent ductules $10 / 10$, epididymis $25 /$ 25 , ovarian follicles $5 / 5$ ) did not demonstrate any TdT reactivity.
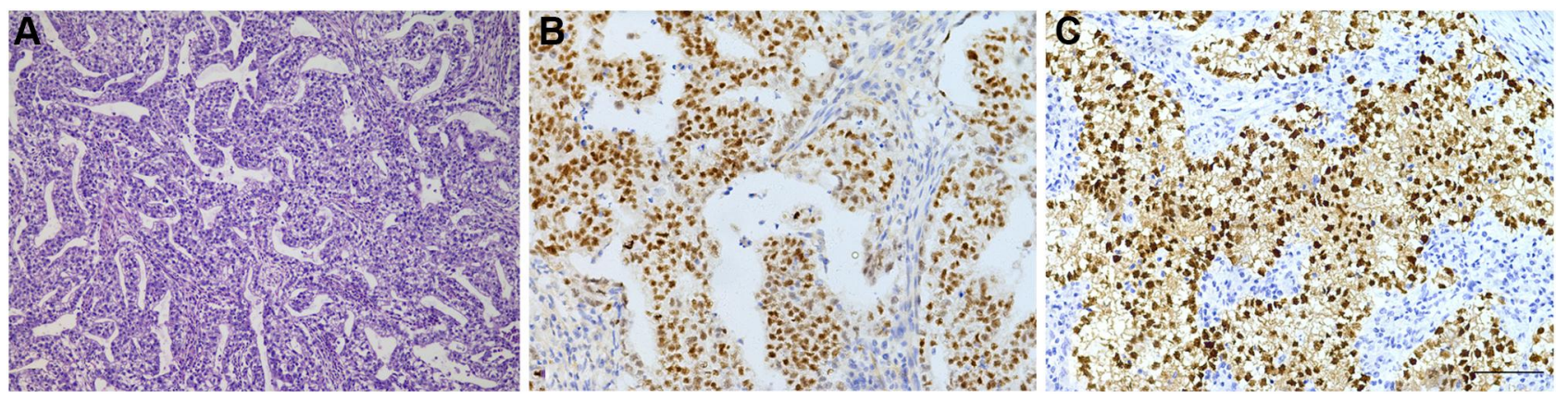

Figure 4 Immunohistochemical staining in EC. ECs demonstrate heterogeneous growth patterns, including solid, complex glandular, or papillary figurations (A). Positivity for TdT in EC (B). The tumors were confirmed by OCT4 (C) (amplification: 10×20, bar=50 $\mu$ m). 

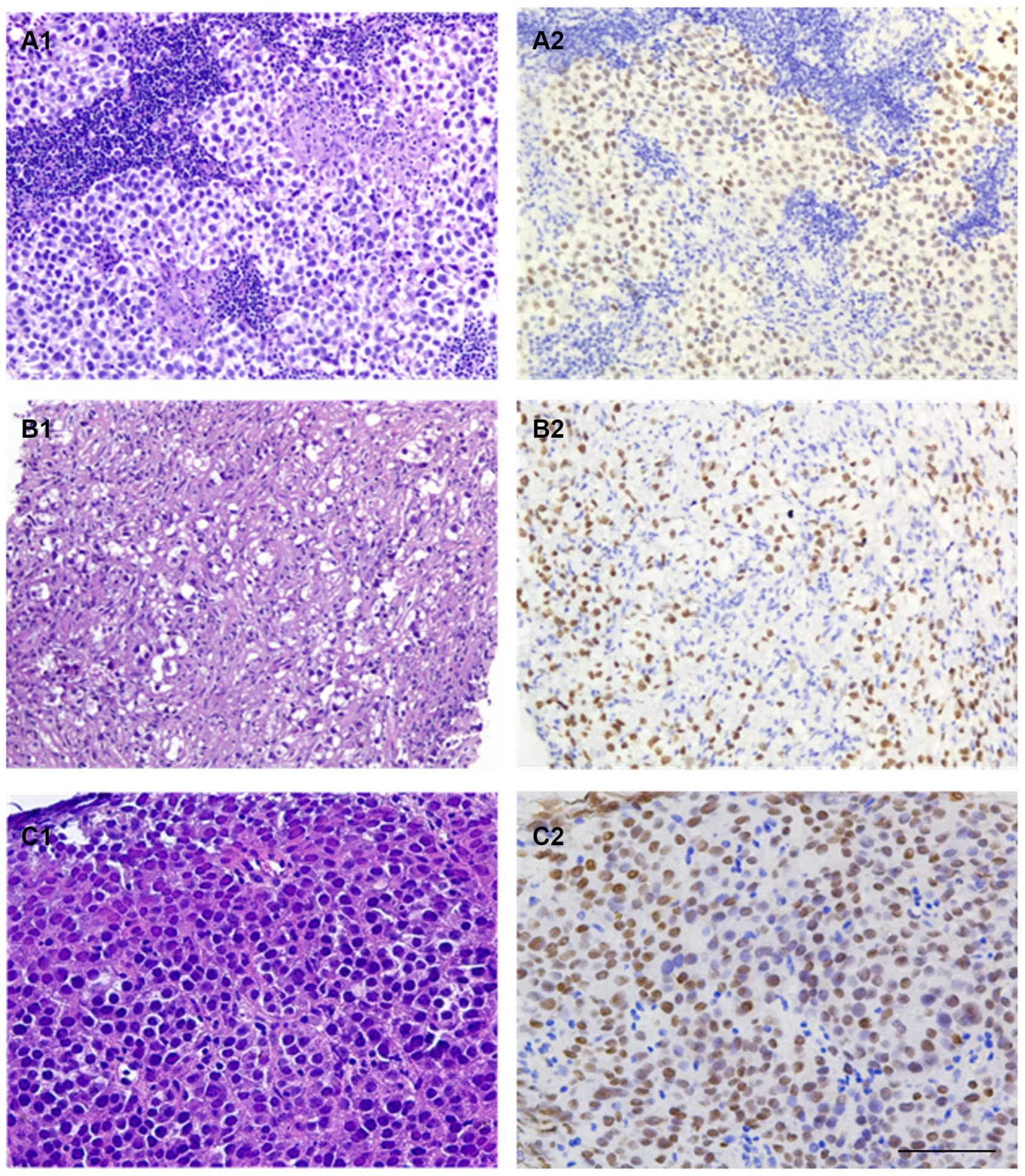

Figure 5 Extragonadal germinomas involving the sellar $(\mathbf{A I})$, mediastinal $(\mathbf{B I})$, and right cervical $(\mathbf{C I})$ regions were obtained through core biopsies. The morphologic features are identical to the testicular or ovarian counterpart; crush artifact and fibrous changes (BI) are not uncommon. All of these tumors express TdT (A2-C2) (amplification: $10 \times 20$, bar $=50 \mu \mathrm{m}$ ).

Comparison of TdT Expression to PLAP, CDII7, OCT4, SALL4, and D2-40

We compared TdT staining with these 5 markers in 6 components in pure or mixed GCTs (seminoma, GCNIS, dysgerminoma, EC, extragonadal germinoma, and YST). As depicted in Table 3, There were no significances between TdT and OCT4 $(P>0.05)$, and they were positive for all components other than the Yolk sac tumor. Identical TdT-positive 
components (seminoma, GCNIS, dysgerminoma, EC, extragonadal germinoma), SALL4 also labeled these tumors without statistical significances $(P>0.05)$, but it stained all YSTs $(P=0.000)$. The expression of PLAP showed positive not only in the overwhelming majority of TdT-positive components $(P>0.05)$ but also in a subset of YSTs $(P=0.000)$. The most of tumor cells were positive for CD117 in seminoma, GCNIS, dysgerminoma, extragonadal germinoma, sharing no significances with TdT $(P>0.05)$, but a fraction of EC $(P=$ $0.000)$ and YST $(P=0.000)$ were positive. As same positivity as TdT $(P>0.05)$, OCT4 stained almost all tumor cells in the components of seminoma, GCNIS, dysgerminoma, extragonadal germinoma but YST. However, few EC components were positive for D2-40 as well $(P=0.006)$, which is distinct from TdT. Concerning the staining intensity, the majority of previously stained slides showed strong positivity for PLAP, OCT4, SALL4, and D2-40, and moderately or weakly positive for CD117. In contrast to that, TdT staining varied from mild to strong staining in our series. However, most cases within 3 years showed a strong positive for TdT. To investigate whether there exists antigen loss over time, we reperformed staining for PLAP, OCT4, SALL4, CD117, and D240 on selected 16 cases ( 12 seminomas, 4 mixed GCTs) filed between January 1, 2011, to June 30, 2014. The intensity of PLAP, OCT4, SALL4, and D2-40 did not become significantly weaker compared to the previously stained tissues. Only a subset of cases disclosed a pronounced attenuation of CD117 staining and hence showed a weak positive pattern. These results investigate that tendency to lose TdT intensity over time on GCT tissues could be its nature per se, regardless of the otherwise objective factors such as tissue fixation, immunohistochemical staining procedures, etc.

\section{Discussion}

It is, as yet, in a largest series of GCTs that we performed TdT staining from multiple centers. These involved cases included both male and female patients. All of these further revealed the concrete expression of TdT in GCTs. Our results showed that TdT is indeed expressed in seminomas, GCNISs, dysgerminomas, ECs, and their counterparts in the mixed GCTs, as well as extragonadal germinomas. The previous studies from both Brobeil, et al, and Jaconi et al showed that seminomas and GCNISs were reproducibly immunoreactive for TdT. ${ }^{17,24}$ Our investigation further confirmed the diagnostic utility of TdT for these tumors. Moreover, for the first time, we also found that TdT also can be positive in pure ECs, and extragonadal germinomas but negative for YSTs, spermatocytic tumors, sex-cord tumors, and the normal tissues in the ovary and testis. No significance by statistical analyses between TdT and otherwise classical markers (PLAP, CD117, OCT4, SALL4, and D2-40) in most of the GCTs, suggests that TdT could be an equivalent diagnostic marker. Therefore, our findings have extended the knowledge of the TdT-positive spectrum. These new findings may contribute to avoid diagnostic pitfalls, particularly when facing the potential diagnosis of GCTs, and the differential diagnosis between YSTs and other GCTs.

Interestingly, in contrast to our and previous studies, data from The Human Protein Atlas (https://www.proteina tlas.org/) and GEPIA (http://gepia.cancer-pku.cn/) exhibited higher mRNA expression of TdT in normal testicular tissues than in their tumors. ${ }^{17,24-27}$ This suggests that other post-transcriptional regulatory mechanisms responsible for this paradox may exist. We did not further investigate this paradox due to the limited experimental conditions in our lab. Moreover, whether TdT proteins really express in the GCT cells is still cryptic. Jaconi et al held that an immunohistochemical nuclear cross-reaction is the possible reason based on their Western blot and mass spectrometry analyses. $^{24}$ However, this is still needed more other upgraded studies.

TdT has the potential to become a new diagnostic marker for seminomas and GCNISs. By TdT immunostaining, one can readily discern scattered malignant tumor cells intercalated between otherwise normal spermatogenic cells in individual seminiferous tubules. Essentially, visualization of such subtle involvements is frequently unintentional and is a strenuous task appreciable solely based on morphology. In our cases, nearly $100 \%$ of GCNIS components were detected by TdT, implying that the excellent recognition of a small malignant transformation using this antibody is not inferior to any other common markers. Only a single case of TdT expression in dysgerminoma has been reported. Our study showed that most of this tumor expressed TdT, except for some archived for a long duration. The expression status of TdT protein in extragonadal germinomas in both male and female patients was also firstly investigated. The robust immunoreactivity en masse prompts the possibility of adding TdT into the regular panel for diagnosis of these diseases. Additionally, $80 \%$ of embryonic carcinomas are also highlighted by TdT staining in male or female cases. Therefore, it seems that TdT has a similar reactive spectrum to OCT4 in germ cell tumors. 
Notwithstanding, an obvious defect in TdT expression in GCTs appeared when we analyzed the immunohistochemical results. Compared to other common markers for GCTs, the long-term paraffin-embedded tumor tissues appear more likely to lose their TdT activity. As described above, the overwhelming majority of components of seminomas (96\%), GCNIs (100\%), ECs (75\%), and dysgerminomas $(88 \%)$ were diffusely and strongly positive for TdT in the past 3 years. However, immunohistochemical intensity significantly became weaker after 3 years. Antigen loss was much more apparent toward the center of the slides, which could potentially be misinterpreted as negative staining. However, the periphery usually retained variable staining. To exclude the possibility that the results were a clone of TdT or the dyeing procedure, we performed TdT staining on several cases using another clone (MX010, Maixin, Fuzhou, China), optimized antigen retrieval or color-developing time, and used other automated immunohistochemistry systems (BOND-MAX, Leica Microsystems, Wetzlar, Germany), but the staining results did not improve. TdT staining was performed again in other reselected paraffined-embedded tissues of these GCTs older than 3 years. PLAP, CD117, OCT4, SALL4, and D2-40 were also re-dyed in these cases. Most of PLAP, OCT4, SALL4, and D2-40 were diffusely and strongly positive other than CD117. The latter is similar to TdT displaying significantly weaker immunostaining in old archived GCT tissues. Nonetheless, all core biopsies of germinomas stored either recently or over 3 years displayed uniform and intensive positivity. The underlying factor may principally rest on terrifically sufficient fixation by formalin because of a very small amount of tissues. Therefore, it is assumed that strong expression of TdT calls for much more adequate formaldehyde fixation in comparison to many other regular antibodies; under equally fixed conditions, the TdT antigen is more prone to lose over time, contributing to weak or false-negative staining. In this study, TdT retention intensity is arranged in descending order as follows: GCNI and extragonadal germinoma $>$ seminoma $>$ dysgerminoma $>$ embryonal carcinoma. The latter tended to significantly lose TdT reactivity on tissues stored over 1.5 years, manifesting subtle nuclear staining. In this sense, it is imperative to be more prudent when interpreting the TdT immunohistochemical results of long-term archived GCT tumors.

The positivity of TdT in germ cell tumors would likely cause problems in our daily practices due to this unknown fact for the vast majority of pathologists. Primary lymphomas account for $1 \%$ to $9 \%$ of testicular neoplasms and $1.5 \%$ of ovarian neoplasms. ${ }^{28,29}$ Although the most common testicular or ovarian lymphomas (eg, diffuse large B cell lymphoma, DLBCL) usually develop in the elderly, primary lymphoma or secondary to systemic lymphohematopoietic malignancies in children or adolescents were also reported, such as Burkitt lymphoma, lymphoblastic lymphoma, DLBCL, and anaplastic large B cell lymphoma. ${ }^{28,30-34}$ Histologically, the seminomas or dysgerminomas and solid ECs can display sheets of tumor cells with pronounced nucleoli that may mimic the lymphomas. In particular, the needle biopsy of extragonadal germinoma or metastatic seminomas/dysgerminomas present with only a limited number of monomorphic round cells. If the primitive round cells are uniformly positive for TdT, that would invite an erroneous interpretation of lymphoblastic lymphoma.

In conclusion, we investigated the expression of TdT in GCTs from the testis, ovary, and extragonadal sites. TdT can be positive in seminomas, GCNISs, dysgerminomas, extragonadal germinomas, and embryonal carcinomas. This indicates that $\mathrm{TdT}$ can be a new sensitive and specific marker for these tumors. However, we firstly emphasized that long-term preserved paraffin-embedded tissues (particularly within 3 years) may be responsible for weak or false-negative staining for TdT due to its antigen loss to a variable degree. Awareness of TdT expression in GCTs is conducive to avoiding misdiagnosis.

\section{Acknowledgments}

All authors review this manuscript and agree to submit it to the International Journal of Surgical Pathology. All authors declare that this manuscript has not been submitted to other journals.

\section{Disclosure}

The authors declare no conflicts of interest.

\section{References}

1. Rijlaarsdam MA, Looijenga LH. An oncofetal and developmental perspective on testicular germ cell cancer. Semin Cancer Biol. 2014;29:59-74. doi:10.1016/j.semcancer.2014.07.003

2. Lobo J, Gillis AJM, Jeronimo C, Henrique R, Looijenga LHJ. Human germ cell tumors are developmental cancers: impact of epigenetics on pathobiology and clinic. Int J Mol Sci. 2019;20:2. doi:10.3390/ ijms20020258

3. Bahrami A, Ro JY, Ayala AG. An overview of testicular germ cell tumors. Arch Pathol Lab Med. 2007;131(8):1267-1280. doi:10.1043/ 1543-2165(2007)131 
4. Ueno T, Tanaka YO, Nagata M, et al. Spectrum of germ cell tumors: from head to toe. Radiographics. 2004;24(2):387-404. doi:10.1148/ rg.242035082

5. Brown J, Friedlander M, Backes FJ, et al. Gynecologic Cancer Intergroup (GCIG) consensus review for ovarian germ cell tumors. Int J Gynecol Cancer. 2014;24(9 Suppl 3):S48-54. doi:10.1097/ IGC.0000000000000223

6. Msaouel P, Bilen MA, Zhang M, Campbell M, Wang J, Tu SM. Recent developments in the management of germ cell tumors. Curr Opin Oncol. 2017;29(3):172-178. doi:10.1097/CCO.0000000000000361

7. Williamson SR, Delahunt B, Magi-Galluzzi C, et al. The world health organization 2016 classification of testicular germ cell tumours: a review and update from the international society of urological pathology testis consultation panel. Histopathology. 2017;70 (3):335-346. doi:10.1111/his.13102

8. Isobe M, Huebner $\mathrm{K}$, Erikson $\mathrm{J}$, et al. Chromosome localization of the gene for human terminal deoxynucleotidyltransferase to region 10q23-q25. Proc Natl Acad Sci US A. 1985;82(17):5836-5840. doi:10.1073/pnas.82.17.5836

9. Dunlap JB, Cascio MJ, Stacey X, Click S, Troxell ML. TdT-positive infiltrate in inflamed pediatric kidney: a potential diagnostic pitfall. Am J Surg Pathol. 2017;41(5):706-716. doi:10.1097/PAS.0000000 000000828

10. Onciu M, Lorsbach RB, Henry EC, Behm FG. Terminal deoxynucleotidyl transferase-positive lymphoid cells in reactive lymph nodes from children with malignant tumors: incidence, distribution pattern, and immunophenotype in 26 patients. Am J Clin Pathol. 2002;118 (2):248-254. doi:10.1309/L3UW-3AE7-L4LB-3QX3

11. Patel KP, Khokhar FA, Muzzafar T, et al. TdT expression in acute myeloid leukemia with minimal differentiation is associated with distinctive clinicopathological features and better overall survival following stem cell transplantation. Mod Pathol. 2013;26 (2):195-203. doi:10.1038/modpathol.2012.142

12. Sur M, AlArdati H, Ross C, Alowami S. TdT expression in Merkel cell carcinoma: potential diagnostic pitfall with blastic hematological malignancies and expanded immunohistochemical analysis. Mod Pathol. 2007;20(11):1113-1120. doi:10.1038/modpathol.3800936

13. Sidiropoulos M, Hanna W, Raphael SJ, Ghorab Z. Expression of TdT in Merkel cell carcinoma and small cell lung carcinoma. Am J Clin Pathol. 2011;135(6):831-838. doi:10.1309/AJCPLCB2Q9QXDZAA

14. Tzorakoleftheraki SE, Iliadis A, Kostopoulos I, Koletsa T. TdT expression in normal and neoplastic sebaceous cells. Histopathology. 2017;71(6):985-988. doi:10.1111/his.13304

15. Zhou J, Xu H, Zeng H, et al. Expression of TdT in myoepithelial cells: investigation in breasts, sweat glands, and salivary lesions emphasizing the never-documented immunohistochemical findings. Int J Surg Pathol. 2020;28(7):711-720. doi:10.1177/1066896920916792

16. Hoang MP, Donizy P, Wu CL, et al. TdT expression is a marker of better survival in merkel cell carcinoma, and expression of B-Cell markers is associated with merkel cell polyomavirus. Am J Clin Pathol. 2020;154(1):38-47. doi:10.1093/ajcp/aqaa017

17. Brobeil A, Wagenlehner F, Gattenlohner S. Expression of terminal deoxynucleotidyl transferase (TdT) in classical seminoma: a potential diagnostic pitfall. Virchows Arch. 2018;472(3):433-440. doi:10.1007/ s00428-018-2313-5

18. World Medical A. World medical association declaration of helsinki: ethical principles for medical research involving human subjects. JAMA. 2013;310(20):2191-2194. doi:10.1001/jama.2013.281053
19. Looijenga LHJ, Van der Kwast TH, Grignon D, et al. Report from the International Society of Urological Pathology (ISUP) consultation conference on molecular pathology of urogenital cancers: IV: current and future utilization of molecular-genetic tests for testicular germ cell tumors. Am J Surg Pathol. 2020;44(7):e66-e79. doi:10.1097/ PAS.0000000000001465

20. Trinh DT, Shibata K, Hirosawa T, et al. Diagnostic utility of CD117, CD133, SALL4, OCT4, TCL1 and glypican-3 in malignant germ cell tumors of the ovary. J Obstet Gynaecol Res. 2012;38(5):841-848. doi:10.1111/j.1447-0756.2011.01798.x

21. Cheng L, Lyu B, Roth LM. Perspectives on testicular germ cell neoplasms. Hum Pathol. 2017;59:10-25. doi:10.1016/j. humpath.2016.08.002

22. Zhou J, Zhang D, Li W, et al. Primary adrenal schwannoma: a series of 31 cases emphasizing their clinicopathologic features and favorable prognosis. Endocrine. 2019;65(3):662-674. doi:10.1007/s12020-019-01992-z

23. Rasmussen OF, Rudbeck L. Immunohistochemistry: A Dako Perspective. In: Lin F, Prichard J, editors. Handbook of Practical Immunohistochemistry: Frequently Asked Questions. New York: Springer; 2015:57-67.

24. Jaconi M, Magni F, Raimondo F, et al. TdT expression in germ cell tumours: a possible immunohistochemical cross-reaction and diagnostic pitfall. J Clin Pathol $</ I>$. 2019;72:536-541. doi:10.1136/ jclinpath-2019-205713

25. Tang Z, Li C, Kang B, Gao G, Li C, Zhang Z. GEPIA: a web server for cancer and normal gene expression profiling and interactive analyses. Nucleic Acids Res. 2017;45(W1):W98-W102. doi:10.1093/nar/gkx247

26. Uhlen M, Fagerberg L, Hallstrom BM, et al. Proteomics. Tissue-based map of the human proteome. Science. 2015;347 (6220):1260419. doi:10.1126/science.1260419

27. Uhlen M, Zhang C, Lee S, et al. A pathology atlas of the human cancer transcriptome. Science. 2017;357:(6352). doi:10.1126/science.aan2507

28. Horne MJ, Adeniran AJ. Primary diffuse large B-cell lymphoma of the testis. Arch Pathol Lab Med. 2011;135(10):1363-1367. doi:10.5858/arpa.2010-0158-RS

29. Yadav BS, George P, Sharma SC, et al. Primary non-Hodgkin lymphoma of the ovary. Semin Oncol. 2020;28(7):e19-30. doi:10.1177/ 1066896920916792

30. Haddy TB, Sandlund JT, Magrath IT. Testicular involvement in young patients with non-Hodgkin's lymphoma. Am J Pediatr Hematol Oncol Fall. 1988;10(3):224-229. doi:10.1097/00043426198823000-00008

31. Vang R, Medeiros LJ, Warnke RA, Higgins JP, Deavers MT. Ovarian non-Hodgkin's lymphoma: a clinicopathologic study of eight primary cases. Mod Pathol. 2001;14(11):1093-1099. doi:10.1038/ modpathol.3880442

32. Lagmay J, Termuhlen A, Fung B, Ranalli M. Primary testicular presentation of ALK-1-negative anaplastic large cell lymphoma in a pediatric patient. J Pediatr Hematol Oncol. 2009;31(5):330-332. doi:10.1097/MPH.0b013e3181979c5e

33. Chong AL, Ngan BY, Weitzman S, Abla O. Anaplastic large cell lymphoma of the ovary in a pediatric patient. $J$ Pediatr Hematol Oncol. 2009;31(9):702-704. doi:10.1097/MPH.0b013e3181acd82b

34. Ahmad SS, Idris SF, Follows GA, Williams MV. Primary testicular lymphoma. Clin Oncol. 2012;24(5):358-365. doi:10.1016/j. clon.2012.02.005 


\section{Publish your work in this journal}

The International Journal of General Medicine is an international, peer-reviewed open-access journal that focuses on general and internal medicine, pathogenesis, epidemiology, diagnosis, monitoring and treatment protocols. The journal is characterized by the rapid reporting of reviews, original research and clinical studies across all disease areas. The manuscript management system is completely online and includes a very quick and fair peer-review system, which is all easy to use. Visit http://www.dovepress.com/ testimonials.php to read real quotes from published authors.

Submit your manuscript here: https://www.dovepress.com/international-journal-of-general-medicine-journal 